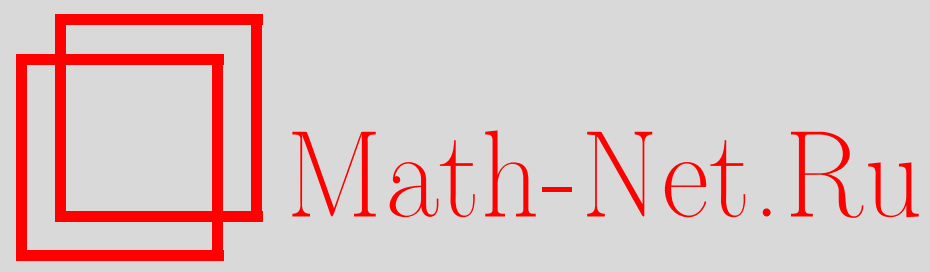

А. И. Созутов, Два признака непростоты группы с сильно вложенной подгруппой и конечной инволюцией, Матем. заметки, 2001, том 69, выпуск 3, 443-453

DOI: https://doi.org/10.4213/mzm516

Использование Общероссийского математического портала Math-Net.Ru подразумевает, что вы прочитали и согласны с пользовательским соглашением http://www . mathnet.ru/rus/agreement

Параметры загрузки:

IP : 54.80 .97 .219

26 апреля 2023 г., $17: 45: 25$

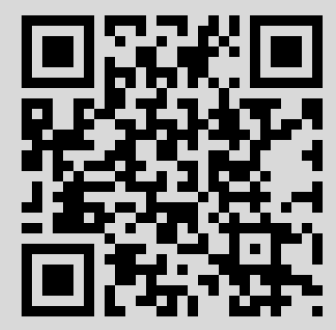




\title{
ДВА ПРИЗНАКА НЕПРОСТОТЫ ГРУППЫ С СИЛЬНО ВЛОЖЕННОЙ ПОДГРУППОЙ И КОНЕЧНОЙ ИНВОЛЮЦИЕЙ
}

\author{
А. И. Созутов
}

Собственная подгруппа $H$ группы $G$ называется сильно вложенной, если $2 \in \pi(H)$ и $2 \notin \pi\left(H \cap H^{g}\right)(\forall g \in G \backslash H)$. Инволюция $i$ группы $G$ назьвается конечной, если $\left|i i^{g}\right|<\infty(\forall g \in G)$. Как известно, строение (локально) конечной группы с сильно вложенной подгруппой в случае, когда силовская 2-подгруппа содержит единственную инволюцию, определяется теоремами Бернсайда и Брауэра-Судзуки. В работе установлены достаточные условия справедливости равенства $m_{2}(G)=1$ и даны два аналога теорем Бернсайда и Брауэра-Судзуки для бесконечной группы $G$ с сильно вложенной подгруппой и конечной инволюцией.

Библиографоия: 18 названий.

В работе обобщаются основные результаты из [1]. Напомним определения. Собственная подгруппа $H$ группы $G$ назьвается сильно вложснной, если $H$ содержит элемент порядка 2 ( инволюиию) и для любого элемента $g \in G \backslash H$ подгруппа $H \cap H^{g}$ не содержит инволюций. Инволюция $i$ групшы $G$ называется конечной, если подгрупшы вида $\left\langle i, i^{g}\right\rangle$ конечны для всех элементов $g \in G$, что равносильно условию $\left|i i^{g}\right|<\infty(g \in G)$. Элемент $a$ бесконечной группы $G$ назьвается почти регулярным, если $\left|C_{G}(a)\right|<\infty$. Группа $G$ (конечная или бесконечная) назьвается группой четного периода, если она содержит инволюцию, и группой нечетного периодав противном случае. Собственная подгрупа $H$ групшы $G$ назьвается сильно изолированной, если она содержит централизатор каждого своего неединичного элемента, т.е. для любого неединичного элемента $g \in G$ из $C_{G}(g) \cap H \neq 1$ следует $C_{G}(g) \leqslant H$, и 2 -изолированной, если это условие вьполняется для $C_{G}(g)$, содержаших инволюции [2]. Подгруппа $H$ групшы $G$ назьвается бесконечно изолированной, если для любого неединичного элемента $h \in H$ из бесконечности $C_{H}(h)$ и четности его периода следует включение $C_{G}(h) \leqslant H[1]$. $F C$-радикалом групшы $G$ назьвается подгруппа $F C(G)=\left\{g \in G|| G: C_{G}(a) \mid<\infty\right\}$.

Строение конечной группы с сильно вложенной подгруппой изучено достаточно детально (см., например, [3]). В случае, когда силовская 2-подгруппа содержит единственную инволюцию, оно определяется теоремами Бернсайда и Брауэра-Судзуки [4], а когда ранг силовской 2 -подгрупшы $\geqslant 2$ - теорией дважды транзитивных групा [3,

Работа выполнена при поддержке Российского фонда фундаментальных исследований, грант № 99-01-00542. 
с. 157-180]. Автором, Н. М. Сучковым и В. Д. Мазуровым [5]-[9] в последнее время получен ряд интересных результатов по бесконечньм кратно транзитивным группам. Теоремы 1, 2 предлагаемой статьи являются некоторыми аналогами теорем Бернсайда и Брауэра-Судзуки [4] для бесконечных групп с сильно вложенной подгруппой и конечной инволюцией и дополняют результаты из [5]-[9].

Теорема 1. Пусть группа $G$ содержит конечную инволюиию, сильно вложсенную бесконечно изолированную подгруппу $H$ и выполняются следующие условия.

1. Централизатор $C_{G}(k)$ некоторой инволюиии $k \in G$ не содержит бесконечных подгрупп, в которых каждый неединичный элемент почти регулярен.

2. Хотя бы одно из пересечений $H \cap H^{g}$, әде $g \in G \backslash H$, бесконечно.

3. Для некоторой инволючии $j \in G \backslash H$ и каждого инвертируемого инволюиией $j$ әлемента $b \in H$ его иентрализатор $C_{G}(b)$ не содерәсится в $H$.

Тогда инволюиии в $G$ сопряэсены, все әлементы $b \in H$, инвертируемые инволюиией $j$, составляют периодическую абелеву нормальную подгруппу $B$ группь $G$, инвертируемую каждой инволючией из $G, u G=C_{G}(B) C_{G}(j)$.

Теорема 2. Пусть группа $G$ содержит конечную инволюиию, сильно вложсенную бесконечно изолированную подгруппу $H$ и выполняются следующие условия.

1. Централизатор $C_{G}(k)$ некоторой инволючии $k \in G$ не содержит бесконечных подгрупп, в которых каждый неединичный әлемент почти регулярен.

2. Хотя бы одно из пересечений $H \cap H^{g}$, әде $g \in G \backslash H$, бесконечно.

3. Существует әлемент $b \in H$, инвертируемый некоторой инволючией $j$ из $G$, иентрализатор $C_{G}(b)$ которого не содерэится в $H$.

Тогда инволюиии в $G$ сопряжены, $B=\left\langle b^{G}\right\rangle$ - периодическая абелева подгруппа, инвертируемая каждой инволючией из $G, u G=C_{G}(B) C_{G}(k)$.

Отметим, что силовские 2-подгрупшы группы $G$ из теорем 1,2 не обязаны быть сопряженньми и изоморфными (пример из п. 3). Заметим также, что в формулировке теоремы 2 инволюция $j$ может быть выбрана из $G \backslash H$, поскольку $H$ сильно вложена и $C_{G}(b) \not H$.

1. Вспомогательные предложения. Нам понадобятся некоторые известные результаты.

ПРЕДЛОЖЕНИЕ 1.1 (лемма Бусаркина). Пусть $G$ - әруппа с конечной инволюиией $i$. Если $C_{G}(i)=\langle i\rangle$, то $G=F \lambda\langle i\rangle$, дде $F$ - периодическая абелева подгруппа из $G$, а инволюиия $i$ инвертирует $F$ [10, предложение 4.2].

ПРЕдЛОЖЕНИЕ 1.2 [1, предложение 12]. 2-группа, обладающая только одной инволюиией, является либо локально ииклической группой (ииклической или квазичиклической), либо обобщенной группой кватернионов (конечной или бесконечной).

ПРЕДЛОЖЕНИЕ 1.3 [11]. Пусть $i$ - почти регулярная конечная инволюиия групnы G. Тогда

1) $G$ - локально конечная группа,

2) $[i, G]$ содержится в $F C$-радикале группь $G$,

3) коммутант $F C$-радикала группы $G$ конечен. 
ПРЕДЛОЖЕНИЕ 1.4 (теорема Бусаркина) [2]. Пусть конечная группа G содержит 2-изолированную подгруппу $H$ четного порядка, вне которой есть инволючии. Тогда $G$ является либо группой Фробениуса, либо $Z T$-группой.

Следующие две леммы хорошо известны и часто будут использоваться в тексте без ссылок.

Лемма 1.1. Пусть $H$ - сильно влохсенная подгруппа группы G. Тогда

1. если $L-$ nодгрупnа групnы $G, L \not \leq H u 2 \in \pi(L \cap H)$, mo noдгруnпа $L \cap H$ сильно вложсна в $L$;

2. $H=N_{G}(H)=N_{G}\left(a^{H}\right)$, где $а$ - любой әлемент четного порядка из $H$.

Лемма 1.2. Пусть $D=\langle i, j\rangle$, əде $i u j-$ инволюиии. Тогда

1. $D=\langle i j\rangle \lambda\langle i\rangle=\langle i j\rangle \lambda\langle j\rangle,(i j)^{i}=(i j)^{j}=j i=(i j)^{-1}$;

2. мнохество $D \backslash\langle i j\rangle$ состоит из инволюций;

3. если $D$ содержит конечную группу диәдра, то она конечна;

4. если $z \in\langle i j\rangle$ инволючия, то $z \in Z(D)$ и в $D$ нет сильно влохсенных подzpynn;

5. если $|i j|$ конечен и нечетен, то любая собственная подгруппа четного порядка из $D$ сильно вложена в $D$;

6. если $|i j|$ конечен и нечетен, то $j=i^{c}$, әде $c \in\langle i j\rangle, c^{2}=i j, u$ для инволюиии $k=і$ выполняются равенства $j=i^{k}, i^{k}=j$.

Доказательства лемм 1.3, 1.4 можно найти в [10], [12] и [5, леммы 2.1, 2.2].

ЛЕмма 1.3. Пусть $G$ - әруппа с сильно вложенной подгруппой $H$ и конечной инволюиией $i$. Тогда

1. все инволюиии в $G$ сопряжены и любые две инволюиии из $H$ сопряжень в $H$;

2. между множеством инволюиий из $Н$ и множеством инволюиий любого правого смежсного класса $\mathrm{Hg}, \mathrm{g} \in \mathrm{G}$, можсно установить взаимно однозначное соответствие, при этом если $i$ - фиксированная инволюиия из $H$, то каждой инволючии $k \in H$ (в том числе и инволючии $i$ ) соответствует единственная инволюиия $j_{k} \in H g$ такая, что $g^{-1} \mathrm{~kg}=j_{k} i j_{k}$;

3. любой әлемент $g \in G$ обладает представлением $g=h j$, где $h \in H, a j-$ некоторая инволюиия;

4. для любой инволюиии $j \in G \backslash H$ в подгруппе $H$ существует множество $M_{j}$ строго вещественных относительно $j$ әлементов той же мощности, что и множество инволюиий из $H$.

ЛЕмма 1.4. Пусть $G$ - әруппа с сильно вложенной подәруппой $H$ и конечной инволючией, $i \in H u j \in G \backslash H$ - произвольные инволюиии, $H_{j}=H \cap H^{j}, T_{j}$ - подгруппа в $\mathrm{H}$, порожденная всеми строго вещественными относительно $j$ әлементами из $H$. Тогда

1. $T_{j} \leqslant H_{j}, H_{j}$ - подгруппа без инволюиий, $\left\langle j, T_{j}\right\rangle=T_{j} \lambda\langle j\rangle u\left\langle j, H_{j}\right\rangle=H_{j} \lambda\langle j\rangle$;

2. в каждом смехнном классе $C_{H}(i) \cdot b$, әде $b \in H$, существует единственный строго вещественный әлемент относительно инволюиии $j$;

3. $H=T_{j} \cdot C_{H}(i)=H_{j} \cdot C_{H}(i)$; 
4. если $b$-неединичный инвертируемый инволющией $j \in G \backslash H$ элемент из $H$, то $C_{H}(b)$ не содерэит инволюиий.

\section{2. Общие леммы и доказательство теоремы 1.}

Лемма 2.1. Пусть выполняются условия 1 и 2 теорем 1,2 и инволюиия из $G \backslash H$. Тогда

1. $H_{j}=T_{j} \lambda C_{j}$, где $C_{j}=C_{H}(j)$;

2. $T_{j}$ - бесконечная абелева периодическая подгруппа, состоящая из всех инвертируемых инволюиией $j$ әлементов из $H$;

3. $C_{j}$ - конечная группа нечетного порядка с ииклическими силовскими подгруппами и любой неединичный әлемент $c \in C_{j}$ почти регулярен в $H$;

4. для любого $p \in \pi\left(C_{j}\right)$ силовские p-подгруппь в $H_{j}$ конечны или черниковские;

5. мноэсество инволюиий в $Н$ бесконечно;

6. если $a \in H_{j}$ и подгруппа $C_{G}(a) \cap H_{j}$ бесконечна, то $a \in T_{j}$.

ДоКАЗАТЕЛЬСТВо. По условию 2 теорем и лемме 1.3 для некоторой инволюции $k \in$ $G \backslash H$ подгруппа $H_{k}=H \cap H^{k}$ бесконечна. Ввиду бесконечной изолированности подгруппы $H$ в $G$ (условия теорем), сопряженности всех инволюций в $G$ (лемма 1.3$)$ и условия 1 теорем подгрупша $C_{H}(k)$ не может быть бесконечной. Следовательно, $H_{k}$ содержит бесконечно много строго вещественных относительно $k$ элементов. По лемме $1.3 H$ содержит бесконечно много инволюций и подгруппы $H_{j}$ и $T_{j}$ бесконечны для произвольной инволюции $j \in G \backslash H$. Из бесконечной изолированности $H$ и отсутствия в $C_{G}(j)$ бесконечных подгрупп, состоящих из почти регулярных элементов (условие 1 теорем), вытекает почти регулярность инволюции $j$ в групше $B=H_{j} \lambda\langle j\rangle$. По предложению 1.3 $B$ - локально конечная группа и $F C$-радикал $F$ групшы $B$ имеет конечный индекс в $B$. Отсюда и из бесконечной изолированности $H$ в $G$ вытекает равенство $C_{F}(j)=1$. Ввиду леммы Бусаркина и конечности инволюции $j$ это означает, что все элементы из $F$ имеют конечные порядки, строго вещественны относительно инволюции $j$, подгрупша $F$ абелева и содержится в $T_{j}$. Отсюда вьводим, что подгрупша $F$ совпадает с $T_{j}$ и не содержит инволюций, при этом $B=F \lambda C_{B}(j)=T_{j} \lambda C_{B}(j)$ (утверждение 2 доказано). Так как $C_{B}(j)$ конечна и ее подгруппа индекса $2 C_{j}=C_{H_{j}}(j)$ не содержит инволюций, то $C_{B}(j)=C_{j} \times\langle j\rangle$ и $H_{j}=T_{j} \lambda C_{j}$ (утверждение 1 доказано).

Далее, если $R$ - элементарная абелева подгруппа ранга 2 из $C_{j}$, то из бесконечности абелевой подгруппы $T_{j}$ вытекает бесконечность $C_{T_{j}}(r)$ для некоторого элемента $r \in R^{\#}$, что противоречит бесконечной изолированности $H$ в $G$. Следовательно, силовские подгруппы в $C_{j}$ циклические (теорема 12.5 .2 из [13]) и каждьй элемент из $C_{j}$ почти регулярен в $B$ (утверждение 3 доказано). В частности, из известной теоремы Блэкберна [14] теперь следует, что силовские $p$-подгрупшы в $T_{j}$ конечны или черниковские для любого $p \in \pi\left(C_{j}\right) \cap \pi\left(T_{j}\right)$ (утверждение 4 леммы).

Как доказано вьше, множество $J$ инволюций подгрупшы $H$ бесконечно. Пусть $a-$ произвольный элемент из $H_{j}$ с бесконечным централизатором $C_{H_{j}}(a)$. Тогда ввиду конечности индекса $\left|H_{j}: T_{j}\right|$ (утверждения 1,3 ) централизатор $D=C_{T_{j}}(a)$ бесконечен. По утверждению $1 a=r b$, где $r \in C_{j}$ и $b \in T_{j}$. Ввиду абелевости $T_{j}$ (утверждение 2 леммы) заключаем, что $D \leqslant C_{H}(r)$ и $r=1$ по утверждению 3 леммы. Следовательно, $a \in T_{j}$ и лемма доказана. 
ЛЕмма 2.2. Если некоторая инволюиия почти регулярна в $G$, то теоремы 1 и 2 верны.

ДоКАЗАТЕЛЬСТВо. В силу леммы 1.3 инволюция $j$ почти регулярна и конечна в $G$. По предложению $1.3 G$ - локально конечная групша с $F C$-радикалом $F$ конечного индекса. Поскольку все инволюции в $G$ сопряжены (лемма 1.3 ), все они почти регулярны и, значит, $F$ - группа без инволюций. По предложению $1.3 F \leqslant[j, G]$, поэтому $G=F \cdot C_{G}(j)$ и $G=F \cdot C_{G}(i)$, где $i$ - инволюция из $H$. Если $C_{F}(j)=1$, то по лемме Бусаркина $F$ - абелева группа, инвертируемая каждой инволюцией из $G$, и $G=F \lambda C_{G}(j)$. По предложению 1.2 силовские 2-подгруппы из $G$ содержат по одной инволюции и являются либо циклическими, либо (обобщенными) группами кватернионов. Ввиду леммы $1.4 T_{j} \leqslant F$ и теорема 2 верна. Далее, $G=F \lambda C_{H}(i)$, поскольку $C_{G}(i)=C_{H}(i)$, и $H=(F \cap H) \lambda C_{H}(i)$. Поэтому подгруппа $F \cap H$ нормальна в $G$, совпадает с $T_{j}$ и инвертируется каждой инволюцией из $G$. Следовательно, теорема 1 также верна, и лемма для случая $C_{F}(j)=1$ доказана.

Пусть $1 \neq r \in F \cap C_{G}(i)$. Тогда в силу бесконечной изолированности подгруппы $H$ имеем $C_{G}(r) \leqslant H$. Следовательно, $|G: H|<\infty$ и по теореме Пуанкаре [15] $H$ содержит нормальную в $G$ подгруппу $D=\cap H^{x}$ конечного индекса. Если $1 \neq r \in C_{D}(j)$, то $\left|C_{D}(r)\right|=\infty$, и ввиду бесконечной изолированности $H$ в $G j \in C_{H}(r) \leqslant H$, противоречие. Следовательно, $C_{D}(j)=1$ и по лемме Бусаркина инволюция $j$ инвертирует $D$. Отсюда вьводим, что $D$ - абелева группа, инвертируемая каждой инволюцией из $G$. Как и выше, заключаем, что силовские 2-подгрупы либо локально циклические, либо (обобщенные) групш кватернионов. Если $b \in D$, то теорема 2 , очевидно, верна. Если $b \in D$ для всех таких $b \in T_{j}$, что $C_{G}(b) \not \leq H$, то верна также и теорема 1.

Пусть теперь $b \in T_{j} \backslash D$ и $r \in C_{G}(b) \backslash H$. По лемме $1.3 r=h k$, где $h \in H, k \in j^{G}$. Поскольку $b \in H \cap H^{r}=H \cap H^{k}$ и $\left|C_{G}(b)\right|=\infty$, по лемме $1.4 b^{k}=b^{-1}$. Но тогда и $b^{h}=b^{-1}$, причем ввиду последнего утверждения леммы 1.4 элемент $b$ инвертируется инволюцией $i \in\langle h\rangle$. Так как по лемме $2.1 i^{H}=i^{T}$ и $T_{j} \leqslant C_{G}(b)$, то каждая инволюция из $H$ инвертирует элемент $b$. Нетрудно убедиться, что подгруппа $B=\left\langle b^{H}\right\rangle$ инвертируется каждой инволюцией из $H$. Действительно, если $x, y$ - произвольные элементы из $H$, инвертируемые каждой инволюцией из $H$, то для любой инволюции $k \in H$ существуют инволюции $v, t \in H$ такие, что $x=k v, y=v t$, и потому $x y=k t$ и $(x y)^{k}=(x y)^{-1}$. Поэтому $B D-$ нормальная в $H$ абелева подгрупп, инвертируемая каждой инволюцией из $H$. Аналогично, любая подгруппа из $G$, инвертируемая каждой инволюцией из $G$, содержится в единственной максимальной нормальной в $G$ подгруппе $T$ с данным свойством.

Рассмотрим подгрупу $L$, порожденную всеми инволюциями из $G$. Понятно, что $L=C_{L}(T) \lambda\langle j\rangle=C_{L}(T) \lambda\langle i\rangle$ и $C_{L}(T) \neq T$. Очевидно, что все инволюции в $L$ сопряжены и по аргументу фраттини $G=L \cdot C_{L}(i)=L \cdot H, i \in H^{\#}, i^{2}=1$. Так как все строго вешественные относительно инволюций элементы группы $G$ содержатся в $L$, то лемму достаточно доказать для групшы $L$. Поэтому можно считать, что уже $L=G=C_{G}(T) \lambda\langle j\rangle=C_{G}(T) \lambda\langle i\rangle$. Ввиду бесконечной изолированности $H$ в $G$ заключаем, что $T \leqslant \cap H^{x}=D$. Далее, централизатор любого элемента нечетного порядка из $H$ содержит $D$ и потому бесконечен. Учитьвая бесконечную изолированность $H$ в $G$ и лемму 2.1 , заключаем, что $C_{H}(k)=1$ и $H \cap H^{k}=T_{k}$ для любой инволюции $k \in G \backslash H$. Отсюда выводим, что в факторгруппе $\bar{G}=G / D$ подгруппа $\bar{H}=H / D$ сильно вложена и 2 -изолирована. Учитьвая строение силовских 2 -подгрупп в $G$ и теорему 
Бусаркина (предложение 1.4), приходим к выводу, что $\bar{G}$ - группа $Ф$ робениуса с неинвариантньп множителем четного порядка и ядром $\bar{R}$. Очевидно, что каждая инволюция из $G$ инвертирует полный прообраз $R$ подгруппы $\bar{R}$ в $G$. Но тогда $R=D$, противоречие. Следовательно, рассматриваемьй случай невозможен. Лемма доказана.

На основании леммы 2.2 в дальнейшем предполагается, что централизаторы инволюций в $G$ бесконечны.

ЛЕмма 2.3. Элемент b из условия 3 теорем 1, 2 инвертирует каждая инволюичя $и з H$.

ДоКАЗАТЕЛЬСТво. По условиям теорем можно выбрать элемент $r \in C_{G}(b) \backslash H$. По лемме $1.3 r=h k$, где $h \in H, k$-инволюция из $G \backslash H$. Имеем $b \in H \cap H^{r}=H \cap H^{k}=H_{k}$. Поскольку $T_{j} \leqslant C_{H}(b)$, то подгруппа $C_{H}(b)$ бесконечна и по лемме $2.1 b \in T_{k}, b^{k}=b^{-1}$. Но тогда и $b^{h}=b^{-1}$. По лемме $1.4 C_{H}(b)$ не содержит инволюций. Поэтому подгруппа $\langle h\rangle$ либо бесконечная циклическая, либо содержит инволюцию $i$, инвертирующую элемент $b$. Пусть верна вторая часть альтернативы. Так как по лемме $2.1 T_{j} \leqslant C_{G}(b)$ и $i^{T}$ - множество всех инволюций из $H$, каждая инволюция из $H$ инвертирует $b$.

Пусть $|h|=\infty$. Как отмечено вьше, централизаторы инволюций в групше $G$ бесконечны. По лемме $2.1 b \in T_{j} \leqslant C_{G}(b)$, поэтому подгруппа $C_{G}(b)$ бесконечна. Далее, по лемме 1.3 инволюция $j$ содержится в некоторой сопряженной с $H$ подгрупе $M$ группы $G$, которая ввиду условий теоремы сильно вложена и бесконечно изолирована, как и подг руппа $H$.

Рассмотрим подгруппу $L=C_{G}(b) \lambda\langle j\rangle$. Очевидно, что элемент $h$ содержится в группе $L$ и $L$ - смешанная группа. Ввиду предложения $1.3 C_{L}(j)$ бесконечен и по условиям теорем существует $1 \neq c \in C_{G}(j) \cap C_{G}(b)$ с бесконечньм централизатором $C_{M}(c)$. Следовательно, $b \in C_{G}(c) \leqslant M$.

Если $T_{j} \leqslant M$, то ввиду леммы 2.1 и очевидного включения $C_{j} \leqslant C_{G}(j) \leqslant M$ вьполняется $H_{j} \leqslant M$. По лемме 1.3 найдется инволюция $v \in G \backslash H$ такая, что $M=H^{v}$ и в силу вьше доказанного $H_{j} \leqslant H_{v}$. Из леммы 2.1 легко следует, что $T_{j} \leqslant T_{v}$. Поэтому условие 3 теорем справедливо для элемента $b$, инволюции $v$ и подгрупшы $M=H^{v}$, при этом инволюция $j \in M$ инвертирует элемент $b$. Как и выше, с помощью леммы 2.1 заключаем, что каждая инволюция из $M$ инвертирует $b$, и в этом случае лемма справедлива. Она верна также при условии $j T_{j} \subseteq M$, поскольку $T_{j} \leqslant\left\langle j T_{j}\right\rangle$. Поэтому можно предполагать, что множество $j T_{j} \backslash M$ непусто и содержит инволюцию $k$, причем $b^{k}=b^{-1}$ (лемма 2.1). В этом случае условие 3 теорем вьполняется для инволюции $k$, элемента $b$ и подгруппы $M$. Используя лемму 2.1 , убеждаемся в том, что каждая инволюция из $M$ инвертирует $b$. Лемма доказана.

Лемма 2.4. Силовские 2-подгруппь из $G$ либо локально ииклические (ииклические или квазициклические), либо обобщенные группы кватернионов (конечные или бесконечные).

ДокАЗАТЕЛЬСТво. По лемме 2.3 любая инволюция из $H$ инвертирует элемент $b$. Отсюда выводим, что в $H$ нет четверных подгрупп, и по предложению 1.2 силовские 2-подгрупшы в $H$ либо локально циклические (циклические или квазициклические), либо обобщенные группы кватернионов (конечные или бесконечные). Таковы они и в $G$, так как из леммы 1.3 следует, что любая силовская 2-подгруппа из $G$ сопряжена с некоторой силовской 2-подгруппой из $H$. Лемма доказана. 
Лемма 2.5. Пусть $B$-множсество всех әлементов из $H$, инвертируемых каждой инволюиией из Н. Тогда справедливы следующие утвержсдения.

1. $B$ - периодическая абелева нормальная в $H$ подгруппа.

2. Если элемент $d \in H$ инвертируется некоторой инволющией $k \in G \backslash H u$ $C_{G}(d) \not \leq H$, то d инвертирует каждая инволюиия из $H u d \in B$.

3. Если элемент $d \in H$ инвертируется некоторой инволюиией $i \in H u C_{G}(d)$ $\not H$, то d инвертирует каждая инволюиия из $H$ u $d \in B$.

ДокАЗАТЕЛЬСТво. 1. Если $x, y \in B$ и $i$ - инволюция из $H$, то $(i x)^{2}=1,(i(x y))^{2}=$ $((i x) y)^{2}=1$; следовательно, $(x y)^{i}=(x y)^{-1}$ и $B$ - подгруппа. Поскольку все инволюции в $H$ конечны (лемма 1.3), то по лемме Бусаркина подгруппа $B$ периодическая и абелева. Наконец, очевидно, что $B$ нормальна в $H$. Утверждение доказано.

2. По лемме 2.3 каждая инволюция из $H$ инвертирует $d$, значит, $d \in B$.

3. Поскольку для $r \in C_{G}(d) \backslash H$ инволюция $k=i^{r} \notin H$ и $d^{k}=d^{-1}$, то $d \in B$ по утверждению 2 леммы. Лемма доказана.

ЛЕмма 2.6. Пусть $k$ - инволюиия из $G \backslash H, A$ - абелева нормальная подгруппа из $H$, содержсащая подгруппу $B, T_{k} \leqslant N_{H}(A), X=T_{k} \cap B \neq T_{k} u X \neq B$. Тогда $X$ нормальна в подгруппе $A T_{k}$ и $A T_{k} / X$ - локально конечная группа Фробениуса с абелевым ядром $A / X$ и локально ииклическим неинвариантным множителем $T_{k} / X$.

ДокАЗАтЕльство. Виду леммы $2.1 V=A T_{k}-$ локально конечная группа, $A \triangleleft V$, $X \triangleleft V, A / X$ и $T_{k} / X$ - абелевы групш. Поэтому достаточно показать, что любые неединичные элементы $\bar{z} \in A / X, \bar{r} \in T_{k} / X$ не перестановочны [16], [17]. Предположим противное. Пусть $\bar{z}, \bar{r}$ таковы, что $\bar{z} \bar{r}=\bar{r} \bar{z}$, т.е. для прообразов $z$ и $r$ данных элементов выполняется равенство $z^{-1} r z=r x$, где $x \in X$. Обозначим $M=\langle X, r\rangle$ и $W=\langle M, z, k\rangle$. Ввиду доказанного вьше $M \triangleleft W, X \triangleleft W$ и в силу перестановочности элементов $\bar{z}, \bar{r}$ и равенства $\bar{r}^{k}=\bar{r}^{-1}$ имеем $W=L \lambda\langle k\rangle, \bar{r} \in Z(L / X)$.

Если $k z k=z^{k} \in H$, то $z \in H \cap H^{k}$. Поскольку $C_{H}(z)$ - бесконечная группа, то по лемме $2.1 z \in T_{k}$, что противоречит выбору элемента $\bar{z}$. Следовательно, $k z k \notin H$ и по лемме $1.3 k z k=h v$, где $h \in H, v$ - инволюция из $G \backslash H$. Поскольку $M^{k z k}=M$ и $\left|C_{H}(M)\right|=\infty\left(M \leqslant T_{k}\right)$, то $M \leqslant H \cap H^{v}$ и $M \leqslant T_{v}$ (лемма 2.1$)$.

Так как $z x \in T_{k}$, то $(r x)^{k}=(r x)^{-1}=r^{-1} x^{-1}$ (лемма 2.1), откуда $r^{z k}=r^{-1} x^{-1}$ или $\left(r^{-1}\right)^{k z k}=r^{-1} x^{-1}$. Аналогично, $(r x)^{v}=r^{-1} x^{-1}$ и поскольку $h=k z k v$, то $\left(r^{-1}\right)^{h}=r x$. Очевидно, $h \in N_{H}(M)$, а из $B \triangleleft H$ (лемма 2.5) и $X=M \cap B$ следует $h \in N_{H}(X)$. Таким образом, $h$ индуцирует на $M / X$ инволютивный автоморфизм и, значит, либо $\langle h\rangle$ содержит инволюцию $i$, либо $|h|=\infty$. В первом случаеввиду лемм $1.3,1.4$ заключаем, что $i$ инвертирует $M$, каждая инволюция из $H$ инвертирует $M$ и $M \leqslant B$. Однако последнее противоречит выбору элемента $r$. Следовательно, $|h|=\infty$.

Рассмотрим подгрупшу $V=\langle h, M, k, v\rangle$. Ввиду предложения 1.3 и конечности инволюции $k$ в $G$ заключаем, что $\left|C_{V}(k)\right|=\infty$. Так как $h, k, v \in N_{V}(M)$ и $|M|<\infty$, то в $C_{V}(k) \cap C_{V}(M)$ найдется неединичный элемент $c$ с бесконечным централизатором $C_{K}(c)$ (условие 1 теорем 1,2 ), где $k \in K=H^{t}, t^{2}=1$ (лемма 1.3). Поэтому $M \leqslant C_{G}(c) \leqslant K$ (т.е. $M \leqslant H \cap H^{t}$ ) и поскольку $C_{H}(M)$ бесконечен, то $M \leqslant T_{t}$ и по лемме $2.1 t$ инвертирует $M$. Теперь рассмотрим подгруппу $Y=\langle V, t\rangle$. Очевидно, что $M \triangleleft Y$, подгруппа 
$Y \cap K \neq Y$ и сильно вложена в $Y$. Применяя леммы $1.1,1.3$, заключаем, что $M$ инвертируется каждой инволюцией из $Y$. Следовательно, $r^{i}=r^{-1}$ для некоторой инволюции $i \in H=K^{t}$. Так как $r \in T_{k}$, то ввиду лемм $1.4,2.1 r$ инвертируется каждой инволюцией из $H$. Но тогда $r \in B$, противоречие.

Таким образом, любые неединичные элементы $\bar{z} \in A / X, \bar{r} \in T_{k} / X$ не перестановочны и $A T_{k} / X$ - локально конечная группа $\Phi$ робениуса [16]. Лемма доказана.

ЛЕмма 2.7. Если выполняются условия теоремы 1, то $B=T_{j}$, подгруппы $B$ и

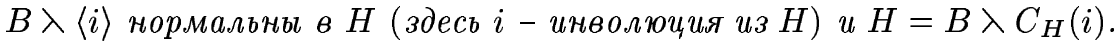

ДокАЗАТЕЛЬСТво. Ввиду условий теоремы 1 и лемм 2.1-2.5 подгруппа $T_{j}$ инвертируется каждой инволюцией из $H$. Поскольку $i^{T_{j}}-$ множество всех инволюций из $H$ (лемма 2.1), то $B=T_{j}$. Очевидно, что $T_{j}=B$ и $B \lambda\langle i\rangle$-характеристические подгруппы группы $H$ и по лемме раттини $H=T_{j} \lambda C_{H}(i)$. Лемма доказана.

Доказательство теоремы 1 завершает

Лемма 2.8. Кажсдая инволюиия әруппь $G$ инвертирует подгруппу $B$ u $G=$ $C_{G}(B) C_{G}(j)$.

ДоКАЗАТЕЛЬСТво. Ввиду лемм 2.1, 2.5, 2.7 достаточно показать, что для каждой инволюции $k$ из $G \backslash H$ выполняется равенство $T_{k}=T_{j}$. Если $T_{k} \leqslant B$, то равенство $T_{k}=T_{j}$ следует из леммы 2.1. Пусть $X=T_{k} \cap T_{j} \neq T_{k}$. Положим в лемме $2.6 A=B$ и $V=B T_{k} / X$. Тогда по лемме $2.6 \mathrm{~V}$ - локально конечная группа $\Phi$ робениуса.

По лемме $2.7 i B$ - множество всех инволюций из $H$ (здесь $i \in H$ - инволюция) и каждая инволюция из $H$ инвертирует $B$ и $X$. По той же лемме $B / X \triangleleft V$ и ввиду равенства $i B=i^{H}$ выполняется включение $T_{k} \leqslant N_{H}(i B)$. Но тогда $\left[T_{k}, i\right] \leqslant B$ и факторгруппа $W / X$ группы $W=\left\langle B, i, T_{k}\right\rangle$ также локально конечная группа Фробениуса. При этом понятно, что $B / X$ - ядро групшы $W$. В силу свойств локально конечных груп $\Phi$ робениуса [16] можно считать, что $\left[i, T_{k}\right] \leqslant X$. Отсюда выводим $i \in N_{G}\left(T_{k}\right)$ и в силу леммы 1.4 инволюция $i$ и каждая инволюция из $H$ инвертирует $T_{k}$. Но это означает $T_{k} \leqslant B$. Следовательно, $T_{k}=B$ и каждая инволюция групшы $G$ инвертирует $B$. Учитывая леммы 1.3 и 2.1 , заключаем, что $B \leqslant \bigcap_{g \in G} H^{g}$ и $B \triangleleft G$.

Далее, если $v$ - произвольная инволюции $G$, то $j v \in C_{G}(B)$. Поэтому все инволюции из $G$ содержатся в подгруппе $L=C_{G}(B) \lambda\langle j\rangle$. Очевидно, что подгрушпа $H \cap L$ сильно вложена в $L$. По лемме 1.3 все инволюции в $L$ сопряжены. По лемме $G=C_{G}(B) C_{G}(j)$. Лемма доказана.

3. Строение подгруппы $C_{G}(b) \lambda\langle j\rangle$ и доказательство теоремы 2. На протяжение всего пункта группа $G$ удовлетворяет условиям теоремы 2 . Пусть $L$ - подгруппа в $G$, порожденная всеми инволюциями из $G$, инвертирующими элемент $b$. Изучим строение этой подгрупшы.

Лемма 3.1. Подгруппа $K=H \cap L$ сильно вложена в $L$ и содержит все инволюичи из $H$.

ДоКАЗАТЕЛЬСТво. По лемме 2.3 все инволюции из $H$ инвертируют элемент $b$. Следовательно, $K$ содержит все инволюции из $H$. Поскольку $j \in L \backslash K$, то $L \neq K$ и $K$ сильно вложена в $L$. Лемма доказана. 
Лемма 3.2. $L=C_{L}(b) \lambda\langle j\rangle$, все инволюиии в $L$ сопряжены и каждая из них инвертирует подгруппу $Z=Z\left(C_{G}(b)\right)$.

ДокАЗАТЕЛЬСТво. Равенство $L=C_{L}(b) \lambda\langle j\rangle$ вытекает непосредственно из определения $L$, а сопряженность инволюций в $L$ - из лемм 3.1 и 1.3. Понятно также, что любой элемент из $Z$, перестановочный с некоторой инволюцией из $L$, перестановочен с любой инволющией из $L$ и принадлежит $Z(L)$. Однако из бесконечной изолированности подгруппы $K$ в $L$, вытекающей из условий теоремы, следует равенство $Z(L)=1$. Значит, любой элемент из $Z$ инвертируется каждой инволюцией из $L$. Лемма доказана.

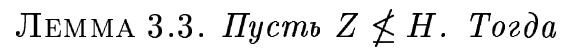

1. $C_{L}(j)-$ конечная группа;

2. $L=F \lambda C_{L}(j)$, әде $F-$ периодическая абелева группа, инвертируемая каждой инволюиией из $L$;

3. $F \cap H=T_{j}$ и в $G$ выполняются условия теоремы 1.

ДокАЗАтЕЛЬство. 1. Пусть $i \in K \cap j^{L}$. Ввиду леммы $3.3 C_{L}(i) \leqslant K$ и если $\left|C_{L}(i)\right|=\infty$, то по условию 2 теоремы 1 пересечение $C_{G}(i) \cap C_{K}(b)$ содержит неединичньй элемент $r$ с бесконечным централизатором $C_{K}(r)$. Тогда ввиду бесконечной изолированности $K$ в $L$ имеем $Z \leqslant C_{L}(r) \leqslant K$, что противоречит условию леммы. Следовательно, $\left|C_{L}(i)\right|<\infty$, что равносильно условию $\left|C_{L}(j)\right|<\infty$. Утверждение доказано.

2. По предложению $1.3 L$ - локально конечная группа с $F C$-радикалом $F$ конечного индекса. Подгрупша $F \cap K$ имеет конечньй индекс в $K$ и так же, как и вьше, из $1 \neq r \in C_{G}(i) \cap F$ следует $Z \leqslant C_{L}(r) \leqslant K$ вопреки условию леммы. Таким образом, $C_{G}(i) \cap F=1$. По лемме Бусаркина $F$ - периодическая абелева группа, состоящая из инвертируемых инволюцией $i$ элементов. Поэтому каждая инволюция из $L$ инвертирует $F$ и $L=F \lambda C_{L}(i)$. Поскольку все инволюции в $L$ сопряжены (леммы $\left.3.1,1.3\right)$, то $L=F \lambda C_{L}(j)$. Утверждение доказано.

3. Учитьвая лемму 2.1 , приходим к вьводу, что $T_{j}=F \cap H$. Но тогда все инволюции из $H$ порождают подгруппу $T_{j} \lambda\langle i\rangle$ (лемма 2.1) и $F \cap H=T_{j}$. Поскольку $Z \leqslant C_{G}\left(T_{j}\right)$ и $Z \not 亡 H$, то вьполняются условия теоремы 1. Лемма доказана.

Лемма 3.4. Если $\left|C_{L}(j)\right|<\infty$, то $C_{G}\left(T_{j}\right) \not \leq H$ и выполняются условия теореMbl 1.

ДоКАЗАТЕЛЬСТВо. Ввиду леммы 3.3 можно считать, что $Z \leqslant K$. По предложению $1.3 L$ - локально конечная группа с $F C$-радикалом $F$ конечного индекса. Пусть $1 \neq r \in C_{L}(j) \cap F$ и $j \in K^{x}=M$. Тогда из бесконечной изолированности подгруппы $M$ следует $C_{G}(r) \leqslant M$. Ввиду конечности индекса $\left|L: C_{L}(r)\right|$ заключаем, что подгруппа $M$ имеет конечный индекс в $L$. Но тогда $|L: K|<\infty$ и по теореме Пуанкаре [15] пересечение всех сопряженных с $K$ подгрупп в $L$ есть нормальная в $L$ подгруппа $D$ конечного индекса. Из лемм 1.4 и 3.3 следует, что $Z \leqslant D$. Поскольку $C_{K}(f)$ бесконечен для всех $f \in F$, то $C_{F}(j)=1$. По лемме Бусаркина $F$ - периодическая абелева подгруппа, причем ввиду предложения $1.3 L=F \lambda C_{L}(j)=F C_{K}(i)$. Следовательно, $K=(F \cap K) \lambda C_{K}(i)$. Отсюда выводим $T_{j} \leqslant F \cap K$ и $F \cap H=T_{j}$. Поскольку $L \not L H$, то $F \not \leq H$ и $C_{G}\left(T_{j}\right) \not \leq H$. Лемма доказана.

Доказательство теоремы 2 завершает 
Лемма 3.5. Кажсдая инволюиия әруппь $G$ инвертирует подгруппу $B$ u $G=$ $C_{G}\left(B_{0}\right) C_{G}(k)$, əде $B_{0}=\left\langle b^{G}\right\rangle$.

ДокАЗАТЕЛЬСТво. Ввиду лемм 2.1, 2.3, 2.5 достаточно показать, что для каждой инволюции $k$ из $G \backslash H$ выполняется включение $B \leqslant T_{k}$. Предположим, что $X=$ $B \cap T_{k} \neq B$ и пусть $A=Z\left(C_{H}(B)\right)$ и $V=A T_{k}$. Пусть $i-$ инволюция из $H$. Поскольку $V=B T_{k} \neq T_{k}$, то ввиду леммы $1.4 V=T_{k} C_{V}(i)$, где $C_{V}(i) \neq 1$. Пусть $1 \neq d \in C_{V}(i)$. Ввиду лемм $1.3,1.4,2.1 d \notin B, d \notin T_{k}$ и $d=z r$, где $1 \neq z \in B, 1 \neq r \in T_{k} \backslash X$. По лемме $2.6 \mathrm{~V} / X$ - локально конечная группа Фробениуса. Так как $i \in N_{H}(X), z^{i}=z^{-1}$, $d^{i}=d$, то $r^{i}=z d, i \in N_{H}(\langle X, r, d, z\rangle)$ и $W=\langle i, X, r, d, z\rangle$ - локально конечная групш. Поскольку $|W:(V \cap W)|=2$ и $(V \cap W) / X$ - группа Фробениуса, a $\langle d X\rangle,\langle r X\rangle$ - ее неинвариантные множители, то некоторая инволюция $v \in W$ нормализует подгрупу $T=\langle r, X\rangle$. Из включения $T \leqslant T_{k}$ и лемм $1.4,2.1$ следует, что $v$ инвертирует $r$. По лемме 2.3 каждая инволюция из $H$ инвертирует элемент $r$. Но тогда $r \in B$, противоречие. Следовательно, $B \leqslant T_{k}$ и каждая инволюция из $G$ инвертирует $B$.

По лемме $2.3 b \in B$ и мы заключаем, что $\left\langle b^{G}\right\rangle=B_{0}$-периодическая абелева подгруппа, инвертируемая каждой инволюцией из $G$. Если $v$ - произвольная инволюции $G$, то $j v \in C_{G}\left(B_{0}\right)$. Поэтому все инволюции из $G$ содержатся в подгрупше $L=C_{G}\left(B_{0}\right) \lambda\langle k\rangle$; здесь $k$ - инволюция из условий теоремы 2 . Очевидно, что подгруппа $H \cap L$ сильно вложена в $L$. По лемме 1.3 все инволюции в $L$ сопряжены. По лемме Фраттини $G=C_{G}\left(B_{0}\right) C_{G}(k)$. Лемма и вместе с ней теорема 2 доказаны.

Примеры груп, иллюстрирующие необходимость условий теорем 1,2 в периодическом случае, приведены в [1]. Дополним их примером смешанной группы.

ПРИмЕР. Ввиду предложения 1.2 произвольная 2-группа ранга 1 содержит единственную инволюцию и является либо локально циклической групшой (циклической или квазищиклической), либо обобщенной группй кватернионов (конечной или бесконечной).

Пусть $S_{1}, S_{2}, \ldots$ - 2-групшы ранга 1 порядка $\geqslant 4$ и $V$ - их свободное произведение с объединенной подгруппой $\langle i\rangle$ порядка 2. Как вытекает из строения групп $S_{1}, S_{2}, \ldots$ и свободных произведений групп с объединенной подгруппой (см., например, [18]), $\langle i\rangle=Z(V)$ и $i-$ единственная инволюция группы $V$.

Рассмотрим сплетение $X=A \imath V=\Phi \lambda V$ абелевой периодической $2^{\prime}$-грушшы $A$ с помощью подгрупшы $V$, где $\Phi$ - база сплетения [15], и пусть $G=X / C_{\Phi}(i)$. Тогда образ $C$ подгруппы $V$ в $G$ изоморфен $V$, инволюция $j=i C_{\Phi}(i)$ единственна в $V$ и инвертирует $F=\Phi / C_{\Phi}(i)$. Поэтому каждая подгрупа $H<G$, содержащая подгрупу $C$, сильно вложена в $G$, а инволюция $j$ конечна в $G$.

Поскольку $C$ - свободное произведение неединичных 2 -групп порядка $\geqslant 4$ с объединенной подгруппой $\langle j\rangle$ порядка 2 , то произвольный элемент $h$ из $C$ либо сопряжен с элементом некоторого сомножителя и $j \in\langle h\rangle$, либо $|h|=\infty$. Пусть справедливо второе. Заметим, что подгруппа $C_{\Phi}(i)$ порождается всевозможными элементами вида $a^{v} a^{v i}$, где $1 \neq a \in A, v \in V$. Пусть $1 \neq a \in A$ и $b=a_{1}^{h_{1}} \cdots a_{k}^{h_{k}}$, где $h_{1}, \ldots, h_{k} \in\langle h\rangle$, $a_{1}, \ldots, a_{k} \in\langle a\rangle$ - различные неединичные элементы. Понятно, что $a \notin C_{\Phi}(i)$ и поскольку $h_{n} \neq h_{m} i$ для любых элементов $h_{n}, h_{m} \in\langle h\rangle$, то $b \notin C_{\Phi}(i)$. Отсюда вьводим, что в факторгруппе $X / C_{\Phi}(i)$ подгрупша $\left\langle h C_{\Phi}(i), a C_{\Phi}(i)\right\rangle$ изоморфна сплетению $\langle a\rangle\langle\langle h\rangle$. Ввиду произвольности $a \in A^{\#}$ заключаем, что $\left\langle F, h C_{\Phi}(i)\right\rangle \simeq A \curlywedge\langle h\rangle$ и $C_{F}(h)=1$. Посколь- 
ку для любого 2-элемента $h \in C C_{F}(h)=1$, то $C_{F}(h)=1$ для каждого неединичного элемента $h \in C$.

Из доказанного выше заключаем, что если $C<H<G$, то $H$ содержит бесконечно много инволюций. Также легко убедиться, что подгруппа $C$ не максимальна в $G$ и потому промежуточная подгруппа $H$ в $G$ существует. Покажем, что подгрупп $H$ бесконечно изолирована в $G$. Действительно, если $h \in H$ и $C_{G}(h)$ четен, то $h \in H \backslash F$ и с точностью до сопряженности в $H$ можно считать, что $h \in C$. В случае, когда $h-$ элемент конечного порядка, сразу получаем $j \in\langle h\rangle$ и $C_{G}(h) \leqslant C \leqslant H$. Пусть $|h|=\infty$ и $r \in C_{G}(h) \backslash C$. Тогда $r=c f$, где $c \in C, 1 \neq f \in F$. Поскольку $j \in C_{G}(h)$, то $r^{j}=c f^{-1} \in C_{G}(h)$ и $1 \neq f^{2}=\left(r^{j}\right)^{-1} r \in C_{G}(h)$. Однако последнее противоречит доказанному выше равенству $C_{F}(h)=1$. Следовательно, $C_{G}(h) \leqslant C \leqslant H$ и $H$ бесконечно изолирована в $G$.

Легко убедиться, что группа $G$ вместе с подгруппой $H$ и произвольным элементом $b \in B^{\#}$ удовлетворяют условиям теорем 1,2 . При этом любая силовская 2-подгруппа из $G$ сопряжена с образом только одной из подгрупп $S_{1}, S_{2}, \ldots$, которые, очевидно, не сопряжены в $G$ и не обязаны быть изоморфными.

\section{СПИСОК ЦИТИРОВАННОЙ ЛИТЕРАТУРЫ}

[1] Измайлов А. Н., Шунков В. П. Два признака непростоты группы с бесконечно изолированной подгруппой // Алгебра и логика. 1982. Т. 21. №6.

[2] Бусаркин В.M. О 2-изолированных подгруппах // Матем. заметки. 1968. Т. 3.№5. C. $497-501$.

[3] Горенстейн Д. Конечные простые группы. М.: Мир, 1985.

[4] Brauer R., Suzuki M. On finite groups of even order whose 2-Sylow group is a quaternion group // Proc. Nat. Acad. Sci. USA. 1959. V. 45. № 12.

[5] Созутов А.И. О некоторых бесконечных группах с сильно вложенной подгруппой // Алгебра и логика (в печати)

[6] Созутов А.И., Сучков Н. М. О некоторых дважды транзитивных группах. Препринт № 17: ИВМ СО РАН, 1998.

[7] Созутов А.И., Сучков Н. М. О некоторых бесконечных кратно транзитивных группах // Международ. алгебраич. семинар, посвященный 70-летию кафедры высшей алгебры МГУ (10-12 февраля 1999 г.). Тез. докл. М., 1999. С. 52.

[8] Созутов А.И., Сучков Н. М. О бесконечных $Z$-группах с заданными стабилизаторами точек // Краснояр. гос. архит.-строит. акад. (Красноярск). Деп. ВИНИТИ № 700-В99 от 09.03.99. М.: ВИНИТИ, 1999.

[9] Мазуров В. Д. О периодических группах с абелевыми централизаторами инволюций // Алгебра и логика (сдана в печать)

[10] Шунков В. П. Мp-группы. М.: Наука, 1990.

[11] Беляев В.В. Группы с почти регулярной инволюцией // Алгебра и логика. 1987. Т. 26. № 5 . C. 531-535.

[12] Шунков В.П. О вложении примарных элементов в группе. Новосибирск: ВО "Наука", 1992.

[13] Холл М. Теория групп. М.: ИЛ, 1962.

[14] Blackburn N. Some remarks on Černikov p-groups. III // J. Math. 1962. V. 6. P. 421-433.

[15] Каргаполов М.И., Мерзляков Ю. И. Основы теории групп. М.: Наука, 1977.

[16] Старостин А. И. О группах Фробениуса // Укр. матем. ж. 1971. Т. 23. № 5. С. 629-639.

[17] Бусаркин В. М., Горчаков Ю. М. Конечные расщепляемые группы. М.: Наука, 1968.

[18] Курош А. Г. Теория групп. М.: Наука, 1967. 